\title{
Non-Invasive Myocardial Infarction Ischemic Area Estimation Through Four-Dimensional Ultrasound
}

\author{
Sydney Q. Clark ${ }^{1}$, Conner C. Earl ${ }^{1,2}$, Joseph M. Gruber ${ }^{2}$, Karthik S. Annamalai ${ }^{2}$, \\ Luke E. Schepers ${ }^{2}$,Craig J. Goergen ${ }^{1,2}$ \\ ${ }^{1}$ Indiana University School of Medicine; ${ }^{2}$ Purdue University, Weldon School of Biomedical \\ Engineering
}

\section{Background/Objective:}

Following myocardial infarction, infarct size and cardiac function are significant predictors of long-term prognosis. Most echocardiography studies rely on two-dimensional analysis for estimation of left ventricular function and electrical activity analysis for estimation of infarct area. Other imaging modalities, such as cardiac magnetic resonance imaging, are limited by time, cost, availability, patient tolerance, and incompatible implantable devices. Using an experimental mouse model of myocardial infarction, we hypothesize that four-dimensional ultrasound offers a possible alternative for easy, quick, and reliable estimation of infarct size.

\section{Methods:}

A cohort of 10 mice underwent four-dimensional cardiac imaging at baseline using a small animal high frequency ultrasound. A thoracotomy was subsequently performed, and a suture placed to ligate the left coronary artery approximately midway down the left ventricle. Sequential four-dimensional ultrasound was performed at six time points over 28 days, following which the mice were euthanized. The hearts were then removed and sent for embedding and sectioning into seven uniform segments stained using both H\&E and Masson's Trichrome.

\section{Results:}

Thus far, we have segmented the imaging and collected end diastolic volume, peak systolic volume, stroke volume, ejection fraction, transmural thickness, and circumferential strain. Additionally, four-dimensional models of the left ventricles have been rendered. Histological embedding, sectioning, and staining is still in progress, and therefore validation against the gold standard is still in process.

\section{Conclusion and Impact:}

Treatment and monitoring of myocardial infarction patients is reliant upon accurate assessment of patient status and prognosis. This study provides initial evidence for the validity of fourdimensional ultrasound as a tool for estimation of myocardial infarction size, providing an alternative to current two-dimensional methods that are less accurate and a more accessible alternative to highly specialized and costly equipment. Improved and accessible imaging methods have the potential to enhance patient care, ultimately improving overall health outcomes. 